\title{
Dias ao Parto de Fêmeas Nelore de um Experimento de Seleção para Crescimento. II - Modelo de Regressão Aleatória ${ }^{1}$
}

\section{Maria Eugênia Zerlotti Mercadante 2, 4, Irineu Umberto Packer 3, 4, Alexander George Razook 2, 4, Claudio Manoel Rodrigues de Melo 3,5 , Joslaine Noely dos Santos Gonçalves Cyrillo², Leopoldo Andrade de Figueiredo ${ }^{2}$}

\begin{abstract}
RESUMO - Os parâmetros genéticos para dias ao parto foram estimados usando um modelo de regressão aleatória, com polinômios ortogonais da idade na monta (em anos) como covariável. Os registros de dias ao parto (4.118) foram provenientes de 926 vacas de três rebanhos Nelore experimentais, sendo os rebanhos seleção e tradicional selecionados para maior peso ao sobreano, e o rebanho controle selecionado para a média do peso ao sobreano. As variâncias genética aditiva e permanente de ambiente foram descritas por uma função polinomial de ordem 4, com nove medidas de erro, resultando em variâncias fenotípica e genética aditiva altas nas idades mais avançadas, principalmente após a 6- monta. As herdabilidades estimadas aumentaram de 0,08 a 0,28 da 1 a à $6 \underline{\text { a }}$ monta. As correlações genéticas foram médias entre o primeiro desempenho e os demais $(0,32$ a 0,66$)$, altas entre os desempenhos adjacentes $(0,98$ a 0,99$)$, e um pouco menores entre os não adjacentes $(0,63$ a 0,98$)$. A seleção para peso não alterou o valor genético médio das vacas dos rebanhos selecionados, entretanto, os valores genéticos médios das vacas do rebanho controle mostraram tendência de queda no decorrer dos anos.
\end{abstract}

Palavras-chave: desempenho reprodutivo, gado de corte, medidas repetidas, função de covariância.

\section{Days to Calving of Nelore Cows from a Selection Experiment for Growth. II - Random Regression Model}

\begin{abstract}
Genetic parameters for days to calving were estimated using a random regression model, with orthogonal polynomials of age at breeding season (in years) as covariable. The records of days to calving $(4,118)$ came from 929 cows from three experimental Nelore herds, been the selection and traditional herds selected for higher yearling weight and the control herd selected for the mean of yearling weight. Genetic and permanent environmental variances were described by a fourth order polynomial function, with 9 measures of error. The phenotypic and additive genetic variances were high in late records, especially after the $6^{\text {th }}$ breeding season. Heritabilities estimates increased from 0.08 to 0.28 , from first up to $6^{\text {th }}$ breeding season. Genetic correlations were moderate between the first and the later records ( 0.32 to 0.66$)$, high between adjacent records ( 0.98 to 0.99$)$, and a little smaller between non adjacent records $(0.63$ to 0.98). The selection for weight did not alter the average breeding value of cows of selection herds, however the average genetic breeding values of control cows declined through the years.
\end{abstract}

Key Words: reproductive performance, beef cattle, repeated records, covariance functions

\section{Introdução}

A Estação Experimental de Zootecnia de Sertãozinho (São Paulo, Brasil) iniciou em 1976 um experimento de seleção com animais Nelore a fim de examinar a resposta à seleção para peso corporal em genótipos de interesse nos trópicos, já que os poucos experimentos de seleção no mundo eram com raças européias. Após 15 anos (progênies de 1981 a 1995), passadas 3,5 gerações de seleção, os machos e as fêmeas do rebanho selecionado são, em média, 17 e $16 \%$ mais pesados à seleção que aqueles do rebanho controle (Razook et al., 1998). Até meados da década de 80 muito se discutiu sobre as consequiências da seleção para peso corporal sobre a eficiência de produção, incluindo o desempenho reprodutivo das matrizes, principalmente pelo aumento no peso adulto advindo dessa seleção. Estudos mais recentes têm mostrado que a seleção para maior peso corporal não apresenta efeitos significativos sobre o desempenho reprodutivo das fêmeas (Meyer et al., 1991; Archer et al., 1998), embora, na maioria deles, os animais tenham sido criados em ambientes menos restritivos que o tropical. 
Dias ao parto tem sido incluído nas avaliações genéticas como um caráter de desempenho reprodutivo das matrizes (Johnston \& Bunter, 1996; CFM, 1999). Registros de dias ao parto durante a vida reprodutiva da matriz são caracterizados como dados longitudinais, ou medidas repetidas no tempo, e geralmente estudados com modelo de repetibilidade que considera todas as medidas como sendo o mesmo caráter (Johnston \& Bunter, 1996; Pereira et al., 2000), ou com modelo uni ou multicaráter, que considera cada medida como um caráter diferente, geralmente os primeiros dois ou três desempenhos (Johnston \& Bunter, 1996; Gressler et al., 2000). O modelo de repetibilidade pode não acomodar heterogeneidade de variâncias e o padrão geral das correlações decrescentes conforme aumenta o intervalo das medidas e, portanto, não ser adequado para descrever as (co)variâncias genéticas dos desempenhos de dias ao parto durante a vida produtiva da matriz. Por outro lado, o modelo multicaráter, com o número de caracteres igual ao número de medidas nas diferentes idades (n), resultaria em análises altamente parametrizadas, com $\mathrm{n}(\mathrm{n}+1) / 2$ componentes de (co)variâncias para descrever a variação genética (Meyer \& Hill, 1997).

Recentemente tem sido reconhecido que modelos de regressão aleatória e suas funções de covariância resultantes são os mais apropriados para análise de dados longitudinais na área de melhoramento genético. Tais modelos acomodam registros repetidos para caracteres que mudam gradualmente através do tempo, não requerendo pressuposições quanto à constância das variâncias e correlações (Meyer, 2000) e tem sido usados, no melhoramento animal, para modelar registros diários de produção de leite durante a lactação em bovinos leiteiros (Jamrozik et al., 1997a, b; Olori, et al., 1999; Rekaya et al., 1999), assim como crescimento corporal em bovinos de corte (Meyer, 1999 e 2000; Albuquerque \& Meyer, 2001), embora a metodologia seja apropriada a outros caracteres em que o fenótipo de um indivíduo é uma função contínua, tal como normas de reação e formas morfológicas, entre outros (Kirkpatrick et al, 1990; Meyer \& Hill, 1997). Modelos de regressão aleatória permitem ajustar uma trajetória aleatória para cada indivíduo como desvios de uma trajetória média da população. A trajetória para uma vaca individual pode ser vista como dois conjuntos de regressões sobre a idade: as regressões fixas para todos os indivíduos pertencentes à mesma subclasse (como ano x rebanho, por exemplo) descrevem a forma geral, enquanto as regressões aleatórias descrevem os desvios genéticos a partir das regressões fixas, permitindo que cada vaca tenha uma forma diferente da trajetória de seus desempenhos em termos genéticos (Jamrozik et al., 1997b).

Análises prévias dos registros de dias ao parto com modelo de repetibilidade, não detectaram diferenças significativas entre os desempenhos de vacas selecionadas para maior peso ao sobreano e de vacas selecionadas para a média deste peso (Mercadante et al., 2002). No presente trabalho serão analisados todos os desempenhos de dias ao parto simultaneamente. Será feita a modelagem da variação genética aditiva e ambiental, usando a técnica de regressão aleatória e o estudo do comportamento genético dos dias ao parto durante a vida produtiva das vacas dos rebanhos selecionados e do controle.

\section{Material e Métodos}

Foram utilizadas informações das vacas pertencentes aos rebanhos experimentais: Nelore Controle (NeC), Nelore Seleção (NeS) e Nelore Tradicional (NeT), da Estação Experimental de Zootecnia de Sertãozinho (EEZS), nascidas entre 1981 e 1996, e participantes das estações de monta de 1983 a 1998. O experimento de seleção iniciou em 1976, com a reorganização e introdução de novas linhagens no rebanho Nelore existente na EEZS, estabelecendose os rebanhos $\mathrm{NeC}, \mathrm{NeS}$ e NeT com 60, 120 e 170 vacas. Foram usados anualmente 4,6 e até 8 touros selecionados de acordo com o diferencial para peso aos 378 dias obtido após prova de desempenho em confinamento, e as fêmeas selecionadas de acordo com o diferencial para peso aos 550 dias, em regime de pasto. Em NeS e NeT foram retidos para reprodução, animais com maior diferencial de seleção e, para $\mathrm{NeC}$, aqueles de diferencial de seleção nulo. Todos os detalhes do processo seletivo foram descritos por Razook et al. (1998).

A variável dias ao parto (DIAP) foi obtida da diferença entre a data do parto e a data da entrada na estação de monta correspondente. Para as vacas que falharam em determinados anos, desde que houvesse o registro de entrada na monta, um registro penalizado foi designado como sendo o maior valor observado de DIAP do grupo contemporâneo (ano do parto + touro em monta) em que ela estava, somado a 21 dias (Johnston \& Bunter, 1996), somente se este grupo 
contemporâneo tivesse mais de quatro observações. As novilhas de reposição foram colocadas em monta natural aos dois anos de idade, junto com o restante das matrizes, por 90 dias, em lotes médios de 25 vacas nos $\mathrm{NeS}$ e $\mathrm{NeT}$ e 15 vacas no $\mathrm{NeC}$ para cada touro. As vacas foram descartadas voluntariamente por falharem dois anos consecutivos, por má habilidade materna, ou por idade avançada, ao redor de 11 anos. Registros provenientes de montas acima de 10 anos foram desconsiderados pelo pequeno número de animais. Um resumo dos dados é apresentado na Tabela 1. A maioria das vacas (56\%) tem mais que três registros de DIAP, com apenas $26 \%$ delas com mais de seis registros.

Os registros de DIAP foram analisados simultaneamente, ajustando um modelo de regressão aleatória com idade na monta (em anos) como a covariável independente através de polinômios ortogonais de ordem quatro $(k=4)$, usando o programa DxMRR

Tabela 1 - Estrutura dos dados, média e desvio-padrão de dias ao parto (DIAP)

Table 1 - Data structure, mean and standard deviation of days to calving (DIAP)

\begin{tabular}{|c|c|}
\hline $\begin{array}{l}\text { Registros } \\
\text { Records }\end{array}$ & 4.118 \\
\hline Animais na análise & 5.754 \\
\hline Animals in analysis & \\
\hline Touros & 125 \\
\hline Sires & \\
\hline Mães & 548 \\
\hline Dams & \\
\hline Animais com registros & 926 \\
\hline Animals with records & \\
\hline Com 1 registro & 155 \\
\hline With 1 record & \\
\hline Com 2 registros & 117 \\
\hline With 2 records & \\
\hline Com 3 registros & 135 \\
\hline With 3 records & \\
\hline Com 4 registros & 105 \\
\hline With 4 records & \\
\hline Com 5 registros & 94 \\
\hline With 5 records & \\
\hline Com 6 registros & 80 \\
\hline With 6 records & \\
\hline Com 7 registros & 59 \\
\hline With 7 records & \\
\hline Com 8 registros & 88 \\
\hline With 8 records & \\
\hline Com 9 registros & 93 \\
\hline With 9 records & \\
\hline Média (dias) & 344 \\
\hline Mean (days) & \\
\hline Desvio-padrão (dias) & 36 \\
\hline Standard deviation (days) & \\
\hline
\end{tabular}

R. Bras. Zootec., v.31, n.4, p.1726-1733, 2002
(Meyer, 1998), que estima as covariâncias entre os coeficientes de regressão aleatória por máxima verossimilhança restrita. As idades na monta foram transformadas em escala logarítmica como sugerido por Meyer (1999), com a finalidade de diminuir a distância entre os registros tomados cedo e tarde na vida do animal, reduzindo o grande efeito que os valores mais afastados da média têm em uma análise de regressão.

O modelo geral pode ser representado por:

$$
\mathrm{y}_{\mathrm{ij}}=\mathrm{F}_{\mathrm{ij}}+\sum_{\mathrm{r}=0}^{\mathrm{k}-1} \beta_{\mathrm{r}} \phi_{\mathrm{r}}\left(\mathrm{a}_{\mathrm{ij}}^{*}\right)+\sum_{\mathrm{r}=0}^{\mathrm{k}-1} \alpha_{\mathrm{ir}} \phi_{\mathrm{r}}\left(\mathrm{a}_{\mathrm{ij}}^{*}\right)+\sum_{\mathrm{r}=0}^{\mathrm{k}-1} \rho_{\mathrm{ir}} \phi_{\mathrm{r}}\left(\mathrm{a}_{\mathrm{ij}}^{*}\right)+\varepsilon_{\mathrm{ij}},
$$

em que: $y_{\mathrm{ij}}$ é o j-ésimo registro do i-ésimo animal, $\mathrm{a}_{\mathrm{ij}}^{*}$ é a idade ao registro padronizada $(-1 \mathrm{a}+1), \phi_{\mathrm{r}}\left(\mathrm{a}_{\mathrm{ij}}^{*}\right)$ é o r-ésimo polinômio de Legendre da idade, $F_{i j}$ é o conjunto de efeitos fixos, $\mathrm{k}$ é a ordem do polinômio, $\beta_{\mathrm{r}}$ são coeficientes de regressão fixa para modelar a média da população, $\alpha_{\text {ir }}$ e $\rho_{\text {ir }}$ são coeficientes de regressão aleatória para modelar os efeitos genético direto e permanente de ambiente, e $\varepsilon_{i j}$ é o erro aleatório associado ao i-ésimo registro do j-ésimo animal. Os primeiros quatro polinômios normalizados são $\sqrt{1 / 2}, \sqrt{3 / 2^{a}} a_{j}^{*},-\sqrt{5 / 8}+\sqrt{45 / 8}\left(a_{j}^{*}\right)^{2} e-\sqrt{63 / 8} a_{j}^{*}+\sqrt{175 / 8\left(a_{j}^{*}\right)^{3}}$, e, assim, um polinômio de ordem 4 envolve potências de idade até 3 , já que o primeiro termo é um escalar (Meyer, 2000). O conjunto de efeitos fixos incluiu o grupo contemporâneo (ano do parto + touro em monta), rebanho de nascimento, estado reprodutivo anterior, e as covariáveis pesos à seleção e na entrada da monta. É pressuposto que $\alpha, \rho$ e $\varepsilon$ apresentam distribuição normal $\operatorname{com} \mathrm{E}(\alpha)=0, \mathrm{E}(\rho)=0$ e $\mathrm{E}(\varepsilon)=0, \operatorname{var}(\alpha)=\mathrm{k}_{\mathrm{A}}, \operatorname{var}(\rho)=\mathrm{k}_{\mathrm{P}}, \operatorname{var}(\varepsilon)=\operatorname{diag}\left\{\sigma_{\mathrm{e}_{\mathrm{j}}}^{2}\right\} \mathrm{e}$ covariâncias nulas entre eles. $\mathrm{k}_{\mathrm{A}}$ e $\mathrm{k}_{\mathrm{P}}$ são matrizes de covariâncias entre os coeficientes de regressão aleatória e $\sigma_{e_{j}}^{2}$ as variâncias de medidas de erro, sendo $\sigma_{e_{j}}^{2}$ dependente do DIAP $j=\{1, \ldots, 9\}$.

Análises prévias foram feitas ajustando modelos com polinômios ortogonais com $\mathrm{k}=1, \ldots, 5$, iguais para as três curvas (média e efeitos aleatórios genético aditivo e permanente de ambiente). $\mathrm{O}$ modelo com polinômio ortogonal de ordem $4(\mathrm{k}=4)$ foi selecionado com base no menor valor do critério de informação de Akaike, que considera a qualidade do ajuste por meio do valor da função de verossimilhança e penaliza para o número de parâmetros do modelo, favorecendo modelos parcimoniosos (Verbeke \& Molenberghs, 2000). O valores genéticos foram preditos somente para os seis primeiros desempenhos de DIAP. 


\section{Resultados e Discussão}

Estimativas de covariâncias e correlações entre os coeficientes de regressão aleatória e seus autovalores associados são apresentados na Tabela 2. A variação devido aos efeitos genético aditivo e permanente de ambiente foi explicada por uma função polinomial de ordem 4, apesar de dois dos coeficientes terem tido contribuição muito pequena para ambos efeitos. Altas correlações entre os coeficientes de regressão fazem com que alguns autovalores sejam negligenciáveis (Albuquerque \& Meyer, 2001).

Geralmente a qualidade do ajuste aumenta com o número de parâmetros da função que descreve a curva. $\mathrm{O}$ valor do $\log$ da função de verossimilhança $(\log \mathrm{L})$ para o modelo com $\mathrm{k}=5$ foi menor que aquele do modelo $\mathrm{k}=4$, mas como o valor do critério de informação de Akaike (CIA) penaliza para o número de parâmetros a serem estimados $(\mathrm{CIA}=-2 \log \mathrm{L}+$ 2q, sendo q o número de parâmetros do modelo), o modelo com $k=4$ teve o menor valor de CIA. Para medidas altamente correlacionadas, há vários autovalores próximos de zero e a informação genética fornecida por $\mathrm{n}$ medidas está totalmente contida por k combinações lineares correspondentes aos maiores autovalores (Meyer \& Hill, 1997).

Com exceção do modelo $\mathrm{k}=1$, as funções de covariâncias estimadas pelos modelos, ajustando polinômios com $\mathrm{k}=2$ a $\mathrm{k}=5$, geraram herdabilidades semelhantes até a 6a medida de DIAP (monta aos 7 anos de idade). Os modelos ajustando polinômios de maiores graus tiveram tendência de aumento excessivo da variância fenotípica e da genética aditiva para as medidas finais (7o, 8o e 9o desempenhos). Meyer (1999), analisando pesos de vacas dos 2 aos 10 anos de idade, com até 9 registros por vaca, ajustando polinômios de Legendre de vários graus, observou que a partir de $\mathrm{k}=3$ as funções de covariâncias, tanto fenotípicas como genéticas, tiveram comportamentos semelhantes, demonstrando que a modelagem é robusta a partir de certa ordem mínima de ajuste.

Os desvios-padrão fenotípico, genético aditivo, permanente de ambiente e residual para todas as idades, gerados a partir da função de covariância estimada, são mostrados na Figura 1. O desvio padrão fenotípico aumentou de 33 dias no início da vida reprodutiva para até 51 dias na última medida (10 anos de idade); o desvio-padrão genético aditivo, de 9 para 43 dias, enquanto que aquele devido ao efeito permanente de ambiente decresceu de 17 para 6 dias do 10 ao 8 o registro, voltando a subir para 16 dias no 90 registro. Os desvios-padrão residuais mantiveram-se mais ou menos constantes, variando de 22 dias aos 10 anos a 29 dias aos 7 anos, sugerindo que um modelo, considerando menos medidas de erro poderia ser usado, o que diminuiria o número de parâmetros a serem estimados. Os valores indicam que até o 60 registro de DIAP, a variância fenotípica parece ter sido consistentemente separada nos seus componentes causais, mas após o 60 registro, a variância fenotípica cresceu muito, provocando aumento excessivo da variância genética e diminuição

Tabela 2 - Estimativas de variâncias (diagonal), covariâncias (abaixo da diagonal) e correlações (acima da diagonal) entre os coeficientes de regressão aleatória da matriz dos coeficientes, e seus autovalores $(\lambda)$, para os efeitos genético aditivo e permanente de ambiente

Table 2 - Estimates of variances (diagonal), covariances (below diagonal) and correlations (above diagonal) between the coefficients of random regression of coefficient matrix, and eigenvalues ( $\lambda$ ), for additive genetic and permanent environmental effects

0

Efeito genético aditivo

Additive genetic effect

$\begin{array}{cc}483,863 & 0,838 \\ 232,329 & 162,784 \\ 84,443 & 76,6030 \\ 34,714 & 30,1674 \\ & \\ & \\ 303,941 & -0,257 \\ -33,977 & 57,469 \\ -73,568 & -38,245 \\ -55,431 & -50,870\end{array}$

Efeito permanente de ambiente

Permanent environmental effect

1

2

$\lambda$




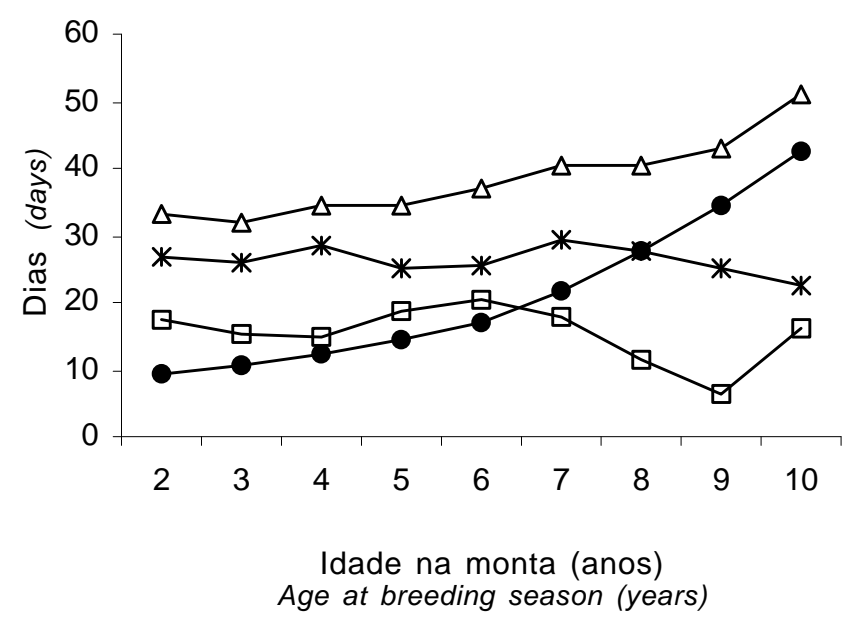

Figura 1 - Desvios-padrão fenotípico $(\Delta)$, residual $\left({ }^{*}\right)$, genético aditivo $(\bullet)$ e permanente de ambiente (ם) estimados para os desempenhos de DIAP até a 10a monta.

Figure 1 - Estimates of the phenotypic $(\Delta)$, residual $\left({ }^{*}\right)$, additive genetic $(\mathbf{\bullet})$ and permanent environmental (口) for the performances of DIAP up to $10^{\text {th }}$ breeding season.

da permanente de ambiente. Valores implausíveis dos componentes de variância nas idades mais avançadas podem ser, provavelmente, devido ao pequeno número de vacas com mais de 6 registros de DIAP (Tabela 1). Entretanto, Meyer (1999) salienta que o fato de usar polinômios ortogonais, que são simétricos, implica que pesos iguais são dados às observações tomadas cedo e tarde na vida, apesar dos últimos registros serem menos representativos do animal, pois são sujeitos a efeitos acumulados de seleção e do ambiente. A transformação logarítmica das idades diminui o espaçamento dos últimos registros, reduzindo a ênfase dada a eles na análise de regressão, e pode contornar o problema, mas não elimina-lo.

Na Figura 2 são apresentadas as herdabilidades (h2) e as frações da variância total devido ao efeito permanente de ambiente (c2). As h2 estimadas aumentaram de 0,08 a 0,28 do 10 ao 6 o desempenho de DIAP, enquanto que o c2 estimado diminuiu de 0,27 a 0,19 neste mesmo período. Mercadante et al. (2002), com os mesmos dados, obtiveram h2 igual a 0,07 para o primeiro desempenho e 0,12 para todos os desempenhos com modelo de repetibilidade, com c 2 igual a 0,09, e Johnston \& Bunter (1996) observaram aumento da 2 do primeiro para o segundo desempenho, de 0,11 para 0,14 , também incluindo registros de vacas que não pariram. Há razões para justificar h2

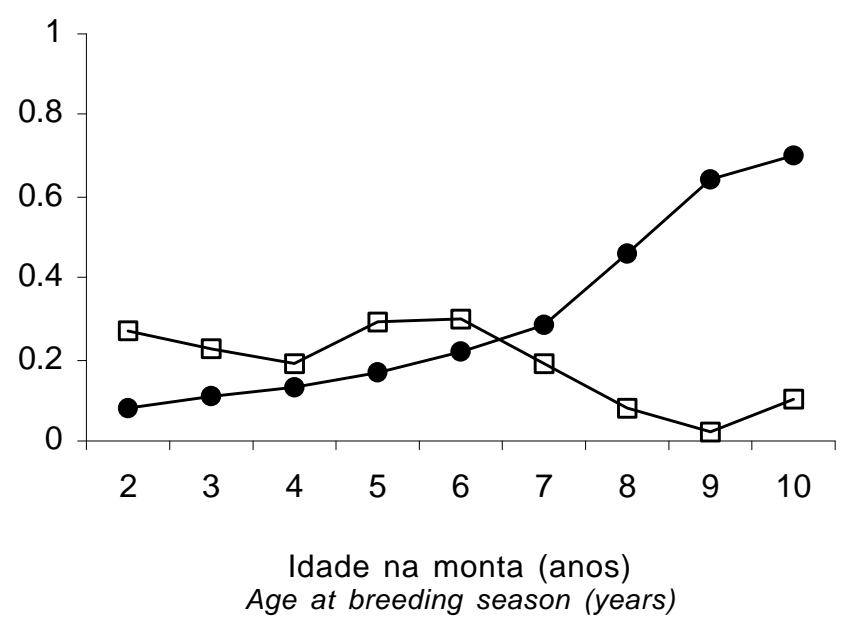

Figura 2 - Herdabilidade (•) e fração da variância total devido ao efeito permanente de ambiente ( $\square$ ) estimadas para os desempenhos de DIAP até a 10 a monta.

Figure 2 - Estimates of heritability (•) and fraction of phenotypic variance due to permanent environmental ( $\square$ ) for the performances of DIAP up to $10^{\text {th }}$ breeding season.

mais altas já que a raça Nelore, ou mais precisamente o rebanho em questão, não tem sido selecionado para reprodução; de modo geral a taxa reprodutiva é baixa e as diferenças entre os reprodutores podem ser mais nítidas nestas populações. Além disso, pode-se supor que em uma estação experimental as condições ambientais sejam mais controladas que em rebanhos comerciais.

As correlações genéticas e fenotípicas entre os desempenhos de DIAP são apresentadas na Tabela 3. Em geral, as correlações tendem a diminuir com o aumento do espaçamento entre os registros. As correlações fenotípicas foram baixas entre os primeiros desempenhos, mesmo entre registros adjacentes, e levemente mais altas após o 3o desempenho. Estes resultados são consistentes com o fato da fêmea estar em crescimento até 5 anos de idade, sendo neste período mais vulneráveis ao estresse ambiental, e, assim, os desempenhos de DIAP mais variáveis. Quando adultas, os desempenhos tendem a ser mais uniformes. As correlações genéticas entre o primeiro desempenho de DIAP e os demais foram as mais baixas, estando de acordo com o fato das fêmeas estarem em crescimento e, portanto, impossibilitadas de expressarem totalmente o potencial genético reprodutivo. Apesar da consistência dos valores de correlação fenotípica e genética aditiva com as fases 
Tabela 3 - Correlações fenotípicas (abaixo da diagonal) e genéticas (acima da diagonal) estimadas para os desempenhos de dias ao parto (DIAP) até 10 anos de idade

Table 3 - Estimates of phenotypics (below diagonal) and genetics (above diagonal) correlations for the performances of days to calving (DIAP) up 10 years old

\begin{tabular}{|c|c|c|c|c|c|c|c|c|c|}
\hline $\begin{array}{l}\text { Anos } \\
\text { Years }\end{array}$ & 2 & 3 & 4 & 5 & 6 & 7 & 8 & 9 & 10 \\
\hline 2 & & 0,63 & 0,62 & 0,66 & 0,64 & 0,57 & 0,48 & 0,39 & 0,32 \\
\hline 3 & 0,23 & & 0,99 & 0,98 & 0,93 & 0,84 & 0,76 & 0,69 & 0,63 \\
\hline 4 & 0,21 & 0,28 & & 0,99 & 0,93 & 0,85 & 0,77 & 0,70 & 0,65 \\
\hline 5 & 0,22 & 0,23 & 0,36 & & 0,98 & 0,92 & 0,86 & 0,80 & 0,75 \\
\hline 6 & 0,21 & 0,20 & 0,35 & 0,48 & & 0,98 & 0,94 & 0,90 & 0,86 \\
\hline 7 & 0,19 & 0,19 & 0,31 & 0,43 & 0,48 & & 0,99 & 0,97 & 0,94 \\
\hline 8 & 0,19 & 0,21 & 0,28 & 0,38 & 0,45 & 0,48 & & 0,99 & 0,98 \\
\hline 9 & 0,16 & 0,23 & 0,23 & 0,28 & 0,35 & 0,42 & 0,55 & & 0,99 \\
\hline 10 & 0,12 & 0,24 & 0,16 & 0,15 & 0,21 & 0,32 & 0,50 & 0,70 & \\
\hline
\end{tabular}

de crescimento e reprodução das matrizes, Albuquerque \& Meyer (2001) salientam que muitos autores têm reportado problemas para modelar covariâncias entre os registros obtidos cedo e tarde na vida do animal, e novamente o pequeno número de observações nas idades extremas, distantes da média, além do ajuste de modelos superparametrizados são colocados como as principais causas.

Nas Figuras 3, 4 e 5 estão plotados os valores genéticos médios das vacas dos três rebanhos experimentais ( $\mathrm{NeC}, \mathrm{NeS}$ e $\mathrm{NeT}$ ) para os seis primeiros desempenhos de DIAP (2 a 7 anos de idade). Os valores genéticos médios das vacas no decorrer dos anos mostraram tendência de diminuição no rebanho controle, sem alteração nos rebanhos selecio-

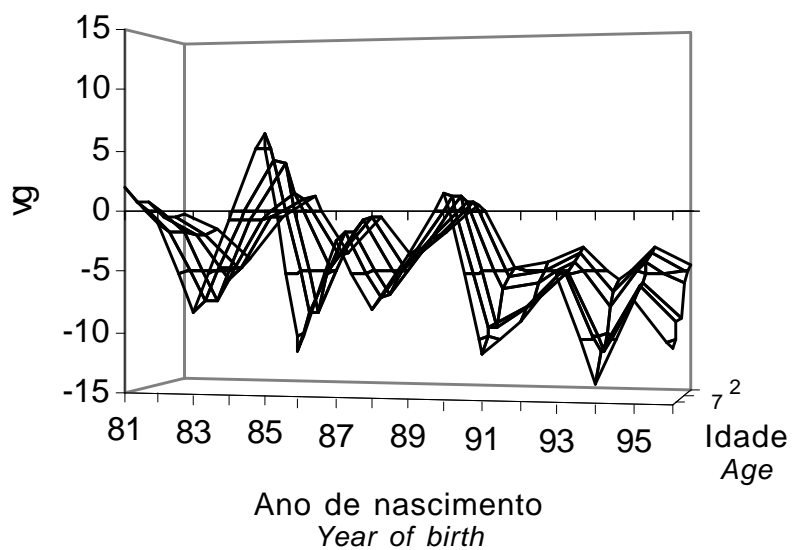

Figura 3 - Valores genéticos médios (dias) para os desempenhos de dias ao parto (DIAP) das vacas do $\mathrm{NeC}$ até a 6a monta, por ano de nascimento.

Figure 3 - Average breeding values (days) for the performances of days to calving (DIAP) of $\mathrm{NeC}$ cows up to $6^{\text {th }}$ breeding season, by year of birth. nados, concordando com Mercadante et al. (2002) que encontraram menores DIAP para $\mathrm{NeC}$, apesar de não serem significativamente diferentes de $\mathrm{NeS}$ e NeT. Analisando os desempenhos longitudinalmente, observa-se menores valores genéticos de DIAP para as vacas do $\mathrm{NeC}$ no decorrer de sua vida produtiva, especialmente após 1986. Vale ressaltar que as vacas nascidas nos últimos anos do experimento não têm desempenho próprio das últimas medidas de DIAP plotadas nos gráficos, ou seja, as vacas nascidas após 1991 tiveram, no máximo, 5 oportunidades.

Se for considerado que as vacas são descartadas pelos mesmos critérios no $\mathrm{NeC} \mathrm{e} \mathrm{NeS,} \mathrm{(o} \mathrm{NeT} \mathrm{é} \mathrm{mais}$ flexível e no início recebeu vacas descartadas por

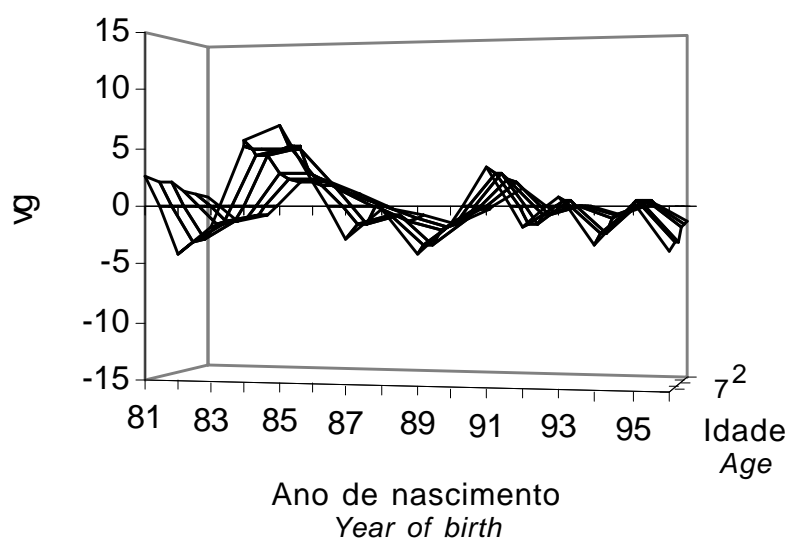

Figura 4 - Valores genéticos médios (dias) para os desempenhos de dias ao parto (DIAP) das vacas do NeS até a 6a monta, por ano de nascimento.

Figure 4 - Average breeding values (days) for the performances of days to calving (DIAP) of NeS cows up to $6^{\text {th }}$ breeding season, by year of birth. 
idade dos outros rebanhos), além do fato daquelas que falharam em determinado ano terem recebido um valor de DIAP maior que suas contemporâneas, os resultados sugerem que as vacas do rebanho controle têm sido mais eficientes no decorrer de sua vida reprodutiva. Entretanto, as vacas do $\mathrm{NeC}$ podem ter sido favorecidas pelo fato dos lotes de monta do rebanho $\mathrm{NeC}$ serem sempre menores que os dos rebanhos $\mathrm{NeS}$ e $\mathrm{NeT}$, em piquetes de mesma dimensão, apesar deste favorecimento já ter sido levado em conta com inclusão do efeito do touro em monta na definição do grupo contemporâneo.

Lembrando do processo seletivo dos três rebanhos ao longo dos 15 anos, ou seja, NeS e NeT selecionados para maiores pesos ao sobreano e $\mathrm{NeC}$ selecionado para a média do peso ao sobreano, tornase difícil explicar que em um rebanho fechado e não selecionado tenha ocorrido mudança na média genética de DIAP ao longo dos anos, como mostra a Figura 3. Uma explicação para isso poderia ser que, devido à limitação de um experimento de seleção quanto ao número de animais, o método não foi totalmente eficiente para separar a mudança genética da ambiental. Ao longo dos 15, anos a mudança ambiental para DIAP, obtida em modelo de repetibilidade, foi igual a $-0,84 \pm 0,32 \mathrm{dia} /$ ano (Mercadante et al., 2002), o que sugere que as vacas

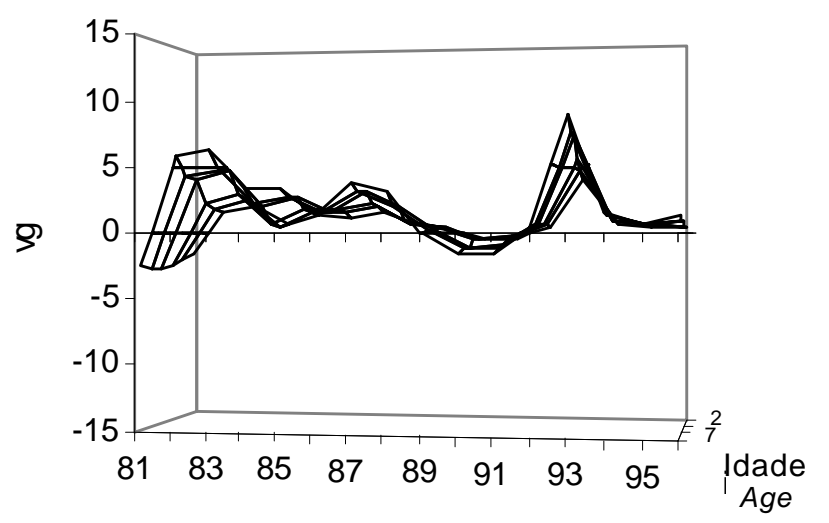

Ano de nascimento Year of birth

Figura 5 - Valores genéticos médios (dias) para os desempenhos de dias ao parto (DIAP) das vacas do NeT até a 6 a monta, por ano de nascimento.

Figure 3 - Average breeding values (days) for the performances of days to calving (DIAP) of $\mathrm{NeT}$ cows up to $6^{\text {th }}$ breeding season, by year of birth. do rebanho controle responderam às mudanças ambientais e os valores genéticos médios mostrados na Figura 3 estão confundidos com efeitos de ambiente. As vacas dos rebanhos selecionados não responderam à mudança ambiental, ou melhor, se não houvesse melhoria nas condições ambientais poderia ter havido aumento do valor fenotípico, e provavelmente genético, do DIAP das vacas $\mathrm{NeS}$ e NeT.

Apesar disso, a aplicação de modelo de regressão aleatória aos registros de DIAP proporcionou uma análise detalhada do comportamento das covariâncias genéticas e do valor genético do desempenho reprodutivo das fêmeas no decorrer de sua vida, o que pode ser oportuno em muitos estudos. Análises adicionais devem ser realizadas em populações maiores, uma vez que os dados foram obtidos em experimento de seleção que por si só tem limitação de número de animais, além de que a metodologia é de certa forma recente, um grande número de modelos e polinômios podem ser testados, e sua aplicação é inédita a dados de reprodução.

\section{Conclusões}

O estudo do comportamento dos dias ao parto ao longo da vida reprodutiva da vaca por meio da técnica de regressão aleatória foi eficiente até o $6 \underline{0}$ desempenho, porém a mudança genética não foi totalmente separada da mudança ambiental.

A seleção para peso não alterou o valor genético para dias ao parto das vacas dos rebanhos selecionados, mas os valores genéticos das vacas do rebanho controle mostraram tendência de queda.

\section{Literatura Citada}

ALBUQUERQUE, L.G.; MEYER, K. Estimates of covariance functions for growth from birth to 630 days of age in Nelore cattle. Journal of Animal Science, v.79, p.2776-2789, 2001.

ARCHER, J.A.; ARTHUR, P.F.; PARNELL, P.F. et al. Effect of divergent selection for yearling growth rate on female reproductive performance in Angus cattle. Livestock Production Science, v.57, p33-40, 1998.

CFM Agro-pecuária. Sumário de Touros Nelore 1999. São José do Rio Preto. 1999. 54p.

GRESSLER, S.L.; BERGMANN, J.A.G.; PEREIRA, C.S. et al. Estudo das associações entre perímetro escrotal e características reprodutivas de fêmeas Nelore. Revista Brasileira de Zootecnia, v.29, p.427-437, 2000.

JAMROZIK, J.; SCHAFFER, L.L. Estimates of genetic parameters for a test day model with random regressions for yield traits of first lactation Holstein. Journal of Dairy Science, v.80, p.762-770, 1997a. 
JAMROZIK, J.; SCHAFFER, L.L. Genetic evaluation of dairy cattle using test day yields and random regression model. Journal of Dairy Science, v.80, p.1217-1226, 1997b.

JOHNSTON, D.J.; BUNTER, K.L. Days to calving in Angus cattle: genetic and environmental effects, and covariances with other traits. Livestock Production Science, v.45, p.13-22, 1996.

KIRKPATRICK, M.; LOFSVOLD, D.; BULMER, M. Analysis of the inheritance, selection and evolution of growth trajectories. Genetics, v.124, p.979-993, 1990.

MERCADANTE, M.E.Z.; PACKER, I.U.; RAZOOK, A.G. et al. Dias ao parto de fêmeas Nelore de um experimento de seleção para peso ao sobreano. Revista Brasileira de Zootecnia, v.31., n.4, p.1715-1725, 2002.

MEYER, K. "DxMRR" - a program to estimate covariance functions for longitudinal data by restricted maximum likelihood. In: WORLD CONGRESS WORLD CONGRESS ON GENETICS APPLIED TO LIVESTOCK PRODUCTION, 6., 1998, Armidale, Australia. Proceedings... Armidale: WCGALP, [1998]. CD-ROM.

MEYER, K. Estimates of genetic and phenotypic covariance functions for postweaning growth and mature weight of beef cows. Journal of Animal Breeding and Genetic, v.116, p.181-205, 1999.

MEYER, K. Random regressions to model phenotypic variation in monthly weights of Australian beef cows. Livestock Production Science, v.65, p.19-38, 2000.

MEYER, K.; HAMMOND, K.; MACKINNON, M.J. et al. Estimates of covariances between reproduction and growth in Australian beef cattle. Journal of Animal Science, v.69, p.3533-3543, 1991.
MEYER, K.M.; HILL, W.G. Estimation of genetic and phenotypic covariance functions for longitudinal or 'repeated' records by restricted maximum likelihood. Livestock Production Science, v.47, p.185-200, 1997.

OLORI, V.; HILL, W.; BROTHERSTONE, S. Estimating variance components for test day milk records by restricted maximum likelihood with a random regression animal model. Livestock Production Science, v.61, p.53-63, 1999.

PEREIRA, E.; ELER, J.P.; FERRAZ, J.B.S. Correlação genética entre perímetro escrotal e algumas características reprodutivas na raça Nelore. Revista Brasileira de Zootecnia, v.29, p.1676-1683. 2000.

RAZOOK, A.G., FIGUEIREDO, L.A.; BONILHA NETO, L. et al. Selection for yearling weight in Nelore and Guzera zebu breeds: selection applied and response in 15 years of progeny. In: WORLD CONGRESS ON GENETICS APPLIED TO LIVESTOCK PRODUCTION, 6., 1998, Armidale, Australia. Proceedings... Armidale: WCGALP, 1998. p.133-136.

REKAYA, R.; CARABAÑO, M.; TORO, M. Use of test-day yields for the genetic evaluation of production traits in Holstein-Friesian cattle. Livestock Production Science, v.34, p.23-34, 1999.

VERBEKE, G.; MOLENBERGHS, G. Linear mixed models for longitudinal data. New York: Springer Verlag, 2000. 568p.

Recebido em: 31/07/01

Aceito em: 17/04/02 